\title{
Informações Editoriais 2012
}

\section{Artigos Publicados - 2012}

\begin{tabular}{lcc}
\hline Ediçães & Autores por Edição & Artigos por Edição \\
\hline Jan/Mar, 2012 & 20 & 6 \\
\hline Abr/Jun, 2012 & 17 & 6 \\
\hline Jul/Set, 2012 & 16 & 6 \\
\hline Out/Dez, 2012 & 21 & 6 \\
\hline Total & 74 & 24 \\
\hline Média & 18,5 & 6 \\
\hline
\end{tabular}

Número de Artigos por Unidade de Federação - 2012

\begin{tabular}{|c|c|c|c|c|c|c|c|c|c|c|}
\hline \multirow{2}{*}{ Edições } & \multicolumn{2}{|c|}{ Jan/Mar } & \multicolumn{2}{|c|}{ Abr/Jun } & \multicolumn{2}{|c|}{ Jul/Set } & \multicolumn{2}{|c|}{ Out/Dez } & \multicolumn{2}{|c|}{ Total } \\
\hline & $\mathrm{Fi}$ & $\mathrm{Fi} \%$ & $\mathrm{Fi}$ & $\mathrm{Fi} \%$ & $\mathrm{Fi}$ & $\mathrm{Fi} \%$ & $\mathrm{Fi}$ & $\mathrm{Fi} \%$ & $\mathrm{Fi}$ & Fi \% \\
\hline Bahia & - & $0 \%$ & 1 & $17 \%$ & - & $0 \%$ & - & $0 \%$ & 1 & $4 \%$ \\
\hline Ceará & 1 & $17 \%$ & - & $0 \%$ & - & $0 \%$ & - & $0 \%$ & 1 & $4 \%$ \\
\hline Distrito Federal & 1 & $17 \%$ & - & $0 \%$ & - & $0 \%$ & - & $0 \%$ & 1 & $4 \%$ \\
\hline Distrito Federal/Goiás & - & $0 \%$ & 1 & $17 \%$ & - & $0 \%$ & - & $0 \%$ & 1 & $4 \%$ \\
\hline Distrito Federal/Goiás & - & $0 \%$ & - & $0 \%$ & - & $0 \%$ & 1 & $17 \%$ & 1 & $4 \%$ \\
\hline Espírito Santo & - & $0 \%$ & 1 & $17 \%$ & 1 & $17 \%$ & - & $0 \%$ & 2 & $8 \%$ \\
\hline Paraná & 1 & $17 \%$ & - & $0 \%$ & - & $0 \%$ & 1 & $17 \%$ & 2 & $9 \%$ \\
\hline Pernambuco/Paraíba & - & $0 \%$ & - & $0 \%$ & - & $0 \%$ & 1 & $17 \%$ & 1 & $4 \%$ \\
\hline $\begin{array}{l}\text { Rio Janeiro/São Paulo/ } \\
\text { Minas Gerais }\end{array}$ & - & $0 \%$ & - & $0 \%$ & - & $0 \%$ & 1 & $17 \%$ & 1 & $4 \%$ \\
\hline Rio Grande do Sul & - & $0 \%$ & 1 & $17 \%$ & - & $0 \%$ & - & $0 \%$ & 1 & $4 \%$ \\
\hline Santa Catarina & 1 & $17 \%$ & 1 & $17 \%$ & 1 & $17 \%$ & - & $0 \%$ & 3 & $13 \%$ \\
\hline São Paulo/Santa Catarina & - & $0 \%$ & - & $0 \%$ & - & $0 \%$ & 1 & $17 \%$ & 1 & $4 \%$ \\
\hline $\begin{array}{l}\text { São Paulo/Santa Catarina/ } \\
\text { Espírito Santo }\end{array}$ & - & $0 \%$ & - & $0 \%$ & 1 & $17 \%$ & - & $0 \%$ & 1 & $4 \%$ \\
\hline São Paulo/Illinois (EUA) & 1 & $17 \%$ & - & $0 \%$ & - & $0 \%$ & - & $0 \%$ & 1 & $4 \%$ \\
\hline São Paulo & - & $0 \%$ & 1 & $17 \%$ & 3 & $50 \%$ & 1 & $17 \%$ & 5 & $21 \%$ \\
\hline Espanha/Rio Grando do Sul & 1 & $17 \%$ & - & $0 \%$ & - & $0 \%$ & - & $0 \%$ & 1 & $4 \%$ \\
\hline Total & 6 & $100 \%$ & 6 & $100 \%$ & 6 & $100 \%$ & 6 & $100 \%$ & 24 & $100 \%$ \\
\hline
\end{tabular}


Número de Autores por Unidade de Federação -2012

\begin{tabular}{|c|c|c|c|c|c|c|c|c|c|c|}
\hline \multirow{2}{*}{ Edições } & \multicolumn{2}{|c|}{ Jan/Mar } & \multicolumn{2}{|c|}{ Abr/Jun } & \multicolumn{2}{|c|}{ Jul/Set } & \multicolumn{2}{|c|}{ Out/Dez } & \multicolumn{2}{|c|}{ Total } \\
\hline & $\mathrm{Fi}$ & $\mathrm{Fi} \%$ & $\mathrm{Fi}$ & $\mathrm{Fi} \%$ & $\mathrm{Fi}$ & $\mathrm{Fi} \%$ & $\mathrm{Fi}$ & $\mathrm{Fi} \%$ & $\mathrm{Fi}$ & $\mathrm{Fi} \%$ \\
\hline Bahia & - & $0 \%$ & 2 & $10 \%$ & - & $0 \%$ & - & $0 \%$ & 2 & $3 \%$ \\
\hline Ceará & 5 & $24 \%$ & - & $0 \%$ & - & $0 \%$ & - & $0 \%$ & 5 & $7 \%$ \\
\hline Distrito Federal & 2 & $10 \%$ & 3 & $14 \%$ & - & $0 \%$ & 3 & $14 \%$ & 8 & $11 \%$ \\
\hline Espírito Santo & - & $0 \%$ & 3 & $14 \%$ & 3 & $14 \%$ & - & $0 \%$ & 6 & $8 \%$ \\
\hline Paraíba & - & $0 \%$ & - & $0 \%$ & - & $0 \%$ & 2 & $10 \%$ & 2 & $3 \%$ \\
\hline Minas Gerais & - & $0 \%$ & - & $0 \%$ & - & $0 \%$ & 1 & $5 \%$ & 1 & $1 \%$ \\
\hline Paraná & 5 & $24 \%$ & - & $0 \%$ & - & $0 \%$ & 4 & $19 \%$ & 9 & $12 \%$ \\
\hline Goiás & - & $0 \%$ & - & $0 \%$ & - & $0 \%$ & 1 & $5 \%$ & 1 & $1 \%$ \\
\hline Pernambuco & - & $0 \%$ & - & $0 \%$ & - & $0 \%$ & 3 & $14 \%$ & 3 & $4 \%$ \\
\hline Rio de Janeiro & - & $0 \%$ & - & $0 \%$ & - & $0 \%$ & 1 & $5 \%$ & 1 & $1 \%$ \\
\hline Rio Grande do Sul & 1 & $5 \%$ & 2 & $10 \%$ & - & $0 \%$ & - & $0 \%$ & 3 & $4 \%$ \\
\hline Rio Grande do Norte & - & $0 \%$ & 1 & $5 \%$ & - & $0 \%$ & - & $0 \%$ & 1 & $1 \%$ \\
\hline Santa Catarina & 4 & $19 \%$ & 3 & $14 \%$ & 7 & $33 \%$ & 2 & $10 \%$ & 16 & $22 \%$ \\
\hline São Paulo & 1 & $5 \%$ & 3 & $14 \%$ & 6 & $29 \%$ & 4 & $19 \%$ & 14 & $19 \%$ \\
\hline Madrid (Espanha) & 1 & $5 \%$ & - & $0 \%$ & - & $0 \%$ & - & $0 \%$ & 1 & $1 \%$ \\
\hline Illinois (EUA) & 1 & $5 \%$ & - & $0 \%$ & - & $0 \%$ & - & $0 \%$ & 1 & $1 \%$ \\
\hline Total & 20 & $100 \%$ & 17 & $100 \%$ & 16 & $100 \%$ & 21 & $100 \%$ & 74 & $100 \%$ \\
\hline
\end{tabular}

Inventário dos Artigos - 2012

\begin{tabular}{lc}
\hline Itens & $\mathbf{2 0 1 2}$ \\
\hline Estoque inicial de artigos em $1^{\circ}$ de janeiro & 73 \\
\hline Número de artigos submetidos & 98 \\
\hline Número de artigos não aprovados no Desk Review & $(74)$ \\
\hline Número de artigos reprovados & $(43)$ \\
\hline Número de artigos retirados & $(3)$ \\
\hline Número de artigos publicados & $(24)$ \\
\hline Número de artigos aceitos para edições seguintes & $(10)$ \\
\hline Numero de artigos em avaliação em 31 de Dezembro & 17 \\
\hline
\end{tabular}


Instituições de Origem dos Autores dos Artigos - 2012

\begin{tabular}{|c|c|c|c|c|c|c|}
\hline \multirow{3}{*}{$\begin{array}{l}\text { UF/IES de origem } \\
\text { BA/UNEB }\end{array}$} & \multicolumn{6}{|c|}{ Edições } \\
\hline & \multirow[t]{2}{*}{ Jan/Mar } & \multirow{2}{*}{$\frac{\text { Abr/Jun }}{1}$} & \multirow[t]{2}{*}{ Jul/Set } & \multirow[t]{2}{*}{ Out/Dez } & \multicolumn{2}{|c|}{ Total } \\
\hline & & & & & 1 & $1 \%$ \\
\hline BA/UFBA & & 1 & & & 1 & $1 \%$ \\
\hline CE/UNIFOR & 1 & & & & 1 & $1 \%$ \\
\hline CE/UFC & 4 & & & & 4 & $5 \%$ \\
\hline DF/UNB & 2 & 3 & & 3 & 8 & $11 \%$ \\
\hline ES/FUCAPE & & 3 & 3 & & 6 & $8 \%$ \\
\hline MG/UFMG & & & & 1 & 1 & $1 \%$ \\
\hline GO/UFG & & & & 1 & 1 & $1 \%$ \\
\hline PB/UFPB & & & & 2 & 2 & $3 \%$ \\
\hline PE/UFPE & & & & 3 & 3 & $4 \%$ \\
\hline PR/PUC & 2 & & & & 2 & $3 \%$ \\
\hline PR/UFPR & 2 & & & 4 & 6 & $8 \%$ \\
\hline PR/UNOPAR & 1 & & & & 1 & $1 \%$ \\
\hline PR/UEL & 1 & & & & 1 & $1 \%$ \\
\hline RJ/UFRJ & & & & 1 & 1 & $1 \%$ \\
\hline RS/UNISC & & 1 & & & 1 & $1 \%$ \\
\hline RS/UNISINOS & & 1 & & & 1 & $1 \%$ \\
\hline RS/FACENSA & 1 & & & & 1 & $1 \%$ \\
\hline SC/UFSC & 1 & & 2 & & 3 & $4 \%$ \\
\hline SC/FURB & 1 & 3 & 3 & 1 & 8 & $11 \%$ \\
\hline SC/F.B.Mendonça & & & 2 & & 2 & $3 \%$ \\
\hline SC/HORUS FAC. & & & & 1 & 1 & $1 \%$ \\
\hline SP/UNINOVE & & & & 1 & 1 & $1 \%$ \\
\hline SP/USP-RP & & & 2 & 2 & 4 & $5 \%$ \\
\hline SP/USP & 1 & 3 & 3 & 1 & 8 & $11 \%$ \\
\hline SP/PUC & & & 1 & & 1 & $1 \%$ \\
\hline USA/UN. ILLINOIS & 1 & & & & 1 & $1 \%$ \\
\hline Espanha/UAM & 1 & & & & 1 & $1 \%$ \\
\hline Outras Instituições & 1 & 1 & & & 2 & $3 \%$ \\
\hline Total & 20 & 17 & 16 & 21 & 74 & $100 \%$ \\
\hline
\end{tabular}




\section{Periodo Médio de Circulação dos Artigos Publicados - 2012}

Vol. 6, n. 1, 2012

\begin{tabular}{|c|c|c|c|c|c|c|c|c|c|}
\hline $\begin{array}{l}\text { N. do } \\
\text { Artigo }\end{array}$ & $\begin{array}{c}\text { Data de } \\
\text { submissão }\end{array}$ & $\begin{array}{l}\text { Avaliado } \\
\text { pelos } \\
\text { Pares }\end{array}$ & $\begin{array}{l}\text { Reformulado } \\
\text { pelos autores }\end{array}$ & $\begin{array}{c}\text { Data da } \\
\text { Aceitação }\end{array}$ & $\begin{array}{c}\text { Data da } \\
\text { Publicação }\end{array}$ & $\begin{array}{c}\text { N. de } \\
\text { dias para } \\
\text { avaliação }\end{array}$ & $\begin{array}{c}\text { N. de } \\
\text { dias para } \\
\text { reformulação }\end{array}$ & $\begin{array}{l}\text { N. de dias para } \\
\text { recomendação } \\
\text { de publicação }\end{array}$ & $\begin{array}{c}\text { N. de dias } \\
\text { entre a } \\
\text { submissão e } \\
\text { publicação }\end{array}$ \\
\hline 1 & $30 / 09 / 2010$ & 04/03/2011 & 06/05/2011 & 07/05/2011 & 27/03/2012 & 154 & 62 & 1 & 537 \\
\hline 2 & 20/12/2010 & 24/08/2011 & 05/09/2011 & $12 / 09 / 2011$ & 27/03/2012 & 244 & 11 & 7 & 457 \\
\hline 3 & $10 / 12 / 2010$ & $10 / 03 / 2011$ & 07/09/2011 & 08/09/2011 & 27/03/2012 & 90 & 177 & 1 & 467 \\
\hline 4 & $21 / 11 / 2010$ & 15/09/2011 & 05/10/2011 & $20 / 11 / 2011$ & 27/03/2012 & 294 & 20 & 45 & 486 \\
\hline 5 & $10 / 12 / 2010$ & $17 / 05 / 2011$ & $28 / 05 / 2011$ & 19/07/2011 & 27/03/2012 & 157 & 11 & 51 & 467 \\
\hline 6 & 09/10/2010 & $28 / 10 / 2010$ & 25/05/2011 & 09/07/2011 & 27/03/2012 & 19 & 207 & 44 & 528 \\
\hline \multicolumn{5}{|c|}{ Média de dias para publicação de artigos por Edição } & & 160,8 & & & 490,3 \\
\hline \multicolumn{6}{|c|}{ Média de meses para publicação de artigos por Edição } & 5,4 & & & 16,3 \\
\hline
\end{tabular}

Vol. 6, n. 2, 2012

\begin{tabular}{|c|c|c|c|c|c|c|c|c|c|}
\hline $\begin{array}{l}\text { N. do } \\
\text { Artigo }\end{array}$ & $\begin{array}{c}\text { Data de } \\
\text { submissão }\end{array}$ & $\begin{array}{l}\text { Avaliado } \\
\text { pelos } \\
\text { Pares }\end{array}$ & $\begin{array}{l}\text { Reformulado } \\
\text { pelos autores }\end{array}$ & $\begin{array}{c}\text { Data da } \\
\text { Aceitação }\end{array}$ & $\begin{array}{c}\text { Data da } \\
\text { Publicação }\end{array}$ & $\begin{array}{c}\text { N. de } \\
\text { dias para } \\
\text { avaliação }\end{array}$ & $\begin{array}{c}\text { N. de } \\
\text { dias para } \\
\text { reformulação }\end{array}$ & $\begin{array}{l}\text { N. de dias para } \\
\text { recomendação } \\
\text { de publicação }\end{array}$ & $\begin{array}{c}\text { N. de dias } \\
\text { entre a } \\
\text { submissão e } \\
\text { publicação }\end{array}$ \\
\hline 1 & $19 / 08 / 2010$ & 06/09/2011 & $22 / 11 / 2011$ & $24 / 11 / 2011$ & 28/06/2012 & 377 & 76 & 2 & 669 \\
\hline 2 & $30 / 12 / 2011$ & $01 / 02 / 2012$ & 26/03/2012 & 27/03/2012 & $28 / 06 / 2012$ & 31 & 55 & 1 & 178 \\
\hline 3 & $28 / 12 / 2010$ & $13 / 01 / 2011$ & $28 / 02 / 2011$ & 28/10/2011 & $28 / 06 / 2012$ & 15 & 45 & 238 & 540 \\
\hline 4 & $13 / 11 / 2010$ & $11 / 03 / 2011$ & $11 / 04 / 2011$ & 26/01/2012 & $28 / 06 / 2012$ & 118 & 30 & 285 & 585 \\
\hline 5 & $08 / 10 / 2010$ & $18 / 01 / 2011$ & $30 / 06 / 2011$ & $19 / 10 / 2011$ & $28 / 06 / 2012$ & 100 & 162 & 109 & 620 \\
\hline 6 & 08/03/2011 & $02 / 04 / 2011$ & $10 / 05 / 2011$ & $31 / 10 / 2011$ & $28 / 06 / 2012$ & 24 & 38 & 171 & 470 \\
\hline \multicolumn{5}{|c|}{ Média de dias para publicação de artigos por Edição } & & 57,6 & & & 510,3 \\
\hline \multicolumn{5}{|c|}{ Média de meses para publicação de artigos por Edição } & & 1,9 & & & 17,0 \\
\hline
\end{tabular}


Vol. 6, n. 3, 2012

\begin{tabular}{cccccccccc}
\hline $\begin{array}{c}\text { N. do } \\
\text { Artigo }\end{array}$ & $\begin{array}{c}\text { Data de } \\
\text { submissão }\end{array}$ & $\begin{array}{c}\text { Avaliado } \\
\text { pelos } \\
\text { Pares }\end{array}$ & $\begin{array}{c}\text { Reformulado } \\
\text { pelos autores }\end{array}$ & $\begin{array}{c}\text { Data da } \\
\text { Aceitação }\end{array}$ & $\begin{array}{c}\text { Data da } \\
\text { Publicação }\end{array}$ & $\begin{array}{c}\text { N. de } \\
\text { dias para } \\
\text { avaliação }\end{array}$ & $\begin{array}{c}\text { N. de } \\
\text { dias para } \\
\text { reformulação }\end{array}$ & $\begin{array}{c}\text { N. de dias para } \\
\text { recomendação } \\
\text { de publicação }\end{array}$ & $\begin{array}{c}\text { N. de dias } \\
\text { entre a } \\
\text { submissão e } \\
\text { publicação }\end{array}$ \\
\hline 1 & $16 / 11 / 2010$ & $06 / 02 / 2011$ & $22 / 02 / 2011$ & $17 / 03 / 2012$ & $14 / 09 / 2012$ & 80 & 16 & 385 & 658 \\
\hline 2 & $14 / 04 / 2011$ & $27 / 11 / 2011$ & $31 / 01 / 2012$ & $02 / 02 / 2012$ & $14 / 09 / 2012$ & 223 & 64 & 2 & 510 \\
\hline 3 & $10 / 06 / 2011$ & $03 / 10 / 2011$ & $30 / 10 / 2011$ & $11 / 01 / 2012$ & $14 / 09 / 2012$ & 113 & 27 & 71 & 454 \\
\hline 4 & $25 / 08 / 2011$ & $08 / 12 / 2011$ & $17 / 02 / 2012$ & $13 / 03 / 2012$ & $14 / 09 / 2012$ & 103 & 69 & 26 & 379 \\
\hline 5 & $09 / 07 / 2011$ & $13 / 10 / 2011$ & $23 / 11 / 2011$ & $27 / 01 / 2012$ & $14 / 09 / 2012$ & 94 & 40 & 64 & 425 \\
\hline 6 & $29 / 08 / 2011$ & $14 / 02 / 2012$ & $17 / 04 / 2012$ & $18 / 06 / 2012$ & $14 / 09 / 2012$ & 165 & 63 & 61 & 375 \\
\hline Média de dias para publicação de artigos por Edição & & & 139,6 & & 466,8 \\
\hline Média de meses para publicação de artigos por Edição & & 4,7 & & 15,6 \\
\hline
\end{tabular}

Vol. 6, n. 4, 2012

\begin{tabular}{|c|c|c|c|c|c|c|c|c|c|}
\hline $\begin{array}{l}\text { N. do } \\
\text { Artigo }\end{array}$ & $\begin{array}{c}\text { Data de } \\
\text { submissão }\end{array}$ & $\begin{array}{l}\text { Avaliado } \\
\text { pelos } \\
\text { Pares }\end{array}$ & $\begin{array}{l}\text { Reformulado } \\
\text { pelos autores }\end{array}$ & $\begin{array}{c}\text { Data da } \\
\text { Aceitação }\end{array}$ & $\begin{array}{c}\text { Data da } \\
\text { Publicação }\end{array}$ & $\begin{array}{c}\text { N. de } \\
\text { dias para } \\
\text { avaliação }\end{array}$ & $\begin{array}{c}\text { N. de } \\
\text { dias para } \\
\text { reformulação }\end{array}$ & $\begin{array}{l}\text { N. de dias para } \\
\text { recomendação } \\
\text { de publicação }\end{array}$ & $\begin{array}{c}\text { N. de dias } \\
\text { entre a } \\
\text { submissão e } \\
\text { publicação }\end{array}$ \\
\hline 1 & $12 / 07 / 2011$ & $15 / 12 / 2011$ & 17/01/2012 & 20/03/2012 & 16/11/2012 & 153 & 32 & 63 & 484 \\
\hline 2 & $06 / 12 / 2011$ & $24 / 04 / 2012$ & $18 / 06 / 2012$ & 27/06/2012 & $16 / 11 / 2012$ & 138 & 54 & 9 & 340 \\
\hline 3 & $16 / 09 / 2011$ & 03/01/2012 & 19/03/2012 & $20 / 04 / 2012$ & $16 / 11 / 2012$ & 107 & 76 & 31 & 420 \\
\hline 4 & $01 / 12 / 2010$ & $12 / 10 / 2011$ & $23 / 01 / 2012$ & 29/06/2012 & $16 / 11 / 2012$ & 311 & 101 & 156 & 705 \\
\hline 5 & $26 / 11 / 2011$ & $11 / 02 / 2012$ & 20/03/2012 & 25/03/2012 & 16/11/2012 & 75 & 39 & 5 & 350 \\
\hline 6 & $12 / 03 / 2011$ & $30 / 06 / 2011$ & 23/11/2011 & $12 / 12 / 2011$ & $16 / 11 / 2012$ & 108 & 143 & 19 & 604 \\
\hline \multicolumn{5}{|c|}{ Média de dias para publicação de artigos por Edição } & & 147,8 & & & 483,8 \\
\hline \multicolumn{6}{|c|}{ Média de meses para publicação de artigos por Edição } & 4,9 & & & 16,1 \\
\hline
\end{tabular}

\begin{tabular}{lcc}
\hline Média de dias para publicação de artigos por Edição em 2012 & 137,1 & 486,8 \\
\hline Média de meses para publicação de artigos por Edição em 2012 & 4,6 & 16,2 \\
\hline
\end{tabular}




\section{Avaliadores Ad Hoc e Instituição de Vínculo - 2012}

\begin{tabular}{|c|c|}
\hline Avaliador & Instituição \\
\hline Adriano Rodrigues & Universidade Federal do Rio de Janeiro \\
\hline Alexandra Vasconcelos Gallon & Universidade Federal do Ceará \\
\hline Alexandre Rivas Rivas & Universidade Federal do Amazonas \\
\hline Alfredo Sarlo Neto & Universidade Federal do Espírito Santo \\
\hline Altair Borgert & Universidade Federal de Santa Catarina \\
\hline Alvaro Augusto Ricardino Filho & Universidade Católica de São Paulo \\
\hline Alvaro Martim Guedes & Universidade Estadual Paulista Julio de Mesquita Filho \\
\hline Amaury José Rezende & Universidade de São Paulo de Ribeirão Preto \\
\hline Ana Carolina Pimentel Duarte da Fonseca & Universidade Federal do Rio de Janeiro \\
\hline Ana Cristina de Faria & Universidade Municipal de São Caetano do Sul \\
\hline Ana Gisbert (Espanha) & Universidade Autonoma de Madrid - Espanha \\
\hline Ana Lúcia Fontes Souza Vasconcelos & Universidade Federal de Pernambuco \\
\hline Ana Maria Roux Cesar & Universidade Presbiteriana Mackenzie \\
\hline Andson Braga Aguiar & Universidade de São Paulo \\
\hline Antonio Artur de Souza & Universidade Federal de Minas Gerais \\
\hline Antonio Benedito Silva Oliveira & Pontifícia Universidade Católica de São Paulo \\
\hline Antonio Carlos Dias Coelho & Universidade Federal do Ceará \\
\hline Antonio Gonçalves de Oliveira & Centro Universitário de Curitiba \\
\hline Antonio Lopo Martinez & Fucape Business School \\
\hline Aridelmo José Campanharo Teixeira & Fucape Business School \\
\hline Artur Roberto do Nascimento & Universidade Estadual de Feira de Santana \\
\hline Bruno Funchal & Fucape Business School \\
\hline Carlos Alberto Diehl & Universidade do Vale do Rio dos Sinos \\
\hline Carlos Alberto Pereira & Universidade de São Paulo \\
\hline Carlos Eduardo Facin Lavarda & Fundação Universidade Regional de Blumenau \\
\hline Carlos Renato Theóphilo & Universidade Estadual de Montes Claros \\
\hline Carlos Roberto Godoy & Universidade de São Paulo - Ribeirão Preto \\
\hline César Augusto Tiburcio Silva & Universidade de Brasília \\
\hline Claudio de Souza Miranda & Universidade de São Paulo de Ribeirão Preto \\
\hline Claudio Parise & Fundação Escola de Comércio Álvares Penteado \\
\hline Daphins Teodoro da Silva Junior & Universidade de São Paulo \\
\hline Dione Olesczuk Soutes & Universidade Estadual do Oeste do Paraná \\
\hline Edgard B. Cornachione Jr & Universidade de São Paulo \\
\hline Elionor Farah Jreige Weffort & Fundação Escola de Comércio Alvares Penteado \\
\hline Ernani Ott & Universidade do Vale do Rio dos Sinos \\
\hline Fabio Gallo Garcia & Fundação Getúlio Vargas - São Paulo \\
\hline
\end{tabular}




\begin{tabular}{|c|c|}
\hline Fábio Moraes da Costa & Fucape Business School \\
\hline Fernando Caio Galdi & Fucape Business School \\
\hline Fernando Dal-Ri Murcia & Universidade Federal de Santa Catarina \\
\hline Fernando de Almeida Santos & Pontifícia Universidade Católica de São Paulo \\
\hline Flávia Zóboli Dalmácio & Universidade de São Paulo \\
\hline Francisco Antonio Bezerra & Fundação Universidade Regional de Blumenau \\
\hline Francisco Carlos Fernandes & Fundação Universidade Regional de Blumenau \\
\hline Gerlando Augusto Sampaio Franco de Lima & Universidade de São Paulo \\
\hline Guillermo Braunbeck & Fundação Instituto de Pesuisas Contábeis, Atuariais e Financeiras \\
\hline Henrique Formigoni & Universidade Presbiteriana Mackenzie \\
\hline Ilse Maria Beuren & Fundação Universidade Regional de Blumenau \\
\hline Irineu Afonso Frey & Universidade Federal de Santa Catarina \\
\hline Ivam Carlos Peleias & Fundação Escola de Comércio Álvares Penteado \\
\hline Jacqueline Veneroso Alves da Cunha & Universidade Federal de Minas Gerais \\
\hline Jeronymo José Libonati & Universidade Federal de Pernambuco \\
\hline Joanilia Neide de Sales Cia & Universidade de São Paulo \\
\hline Joanilia Neide de Sales Cia. & Universidade de São Paulo \\
\hline Jorge de Souza Bispo & Receita Federal do Brasil \\
\hline Jorge Eduardo Scarpin & Fundação Universidade Regional de Blumenau \\
\hline Jorge Katsumi Nyama & Universidade de Brasília \\
\hline Jose Alexandre Magrini Pigatto & Universidade Federal de Santa Maria \\
\hline José Alonso Borba & Universidade Federal de Santa Catarina \\
\hline José Alonso Borba & Universidade Federal de Santa Catarina \\
\hline José Carlos Tiomatsu Oyadomari & Universidade Presbiteriana Mackenzie \\
\hline José Ellias Feres de Almeida & Universidade Federal do Espírito santo \\
\hline José Maria Dias Filho & Universidade Federal da Bahia \\
\hline José Moreira Silva Neto & Universidade Federal de Rondônia \\
\hline José Roberto Kassai & Universidade de São Paulo \\
\hline Joshua Onome Inoniama & Universidade Presbiteriana Mackenzie \\
\hline Lauro Brito de Almeida & Universidade Federal do Paraná \\
\hline Lino Martins da Silva & Universidade Estadual do Rio de Janeiro \\
\hline Luiz Alberton & Universidade Federal de Santa Catarina \\
\hline Luiz Eduardo Afonso & Universidade de São Paulo \\
\hline Luiz Jurandir Simões & Universidade de São Paulo \\
\hline Luiza Maria Bessa Rebelo & Universidade Federal do Amazonas \\
\hline Maisa de Souza Ribeiro & Universidade de São Paulo - Ribeirão Preto \\
\hline Marcelle Colares Oliveira & Universidade de Fortaleza \\
\hline
\end{tabular}




\begin{tabular}{|c|c|}
\hline Marcelo Alvaro da Silva Macedo & Universidade Federal do Rio de Janeiro \\
\hline Marcelo Sanches Pagliarussi & Fucape Business School \\
\hline Marcia Maria dos Santos Bortolocci Espejo & Universidade Federal do Paraná \\
\hline Marcia Martins Mendes De Luca & Universidade Federal do Ceará \\
\hline Marcos Antonio de Souza & Universidade do Vale do Rio dos Sinos \\
\hline Marcus Vinicius Veras Machado & Universidade Federal do Ceará \\
\hline Mariana Simões Ferrazdo Amaral Fregonesi & Universidade de São Paulo \\
\hline Martinho Mauricio Gomes de Ornelas & Universidade Federal da Paraíba \\
\hline Milanez Silva de Souza & Universidade Federal do Amazonas \\
\hline Monica Sionara Schpallir Calijuri & Universidade Presbiteriana Mackenzie \\
\hline Osmar Siena Siena & Universidade Federal de Rondônia \\
\hline Patrícia Siqueira Varela & Fundação Universidade Regional de Blumenau \\
\hline Poueri do Carmo Mario & Universidade Federal de Minas Gerais \\
\hline Raimundo Nonato Rodrigues & Universidade Federal de Pernambuco \\
\hline Renê Coppe Pimentel & Fucape Business School \\
\hline Rodrigo de Souza Goncalves & Universidade de Brasilia \\
\hline Rogério João Lunkes & Universidade Federal de Santa Catarina \\
\hline Romildo de Oliveira Moraes & Universidade Estadual de Maringá \\
\hline Romualdo Douglas Colauto & Universidade Federal do Paraná \\
\hline Sandra Maria dos Santos & Universidade Federal do Ceará \\
\hline Sérgio de ludícibus & Pontifícia Universidade Católica de São Paulo \\
\hline Silvia Pereira de Castro Casa Nova & Universidade de São Paulo \\
\hline Silvio Hiroshi Nakao & Universidade de São Paulo \\
\hline Sirlei Lemos & Universidade Federal de Uberlândia \\
\hline Solange Garcia dos Reis & Universidade de São Paulo de Ribeirão Preto \\
\hline Sonia Maria da Silva Gomes & Universidade Federal da Bahia \\
\hline Umbelina Cravo Texeira Lagioia & Universidade Federal de Pernambuco \\
\hline Valcemiro Nossa & Fucape Business School \\
\hline Vera Maria Rodrigues Ponte & Universidade Federal do Ceará \\
\hline Vilma Geni Slomski & Fundação Escola de Comércio Alvares Penteado \\
\hline Vinicius Aversari Martins & Universidade de São Paulo de Ribeirão Preto \\
\hline Waldemar Antonio da Rocha de Souza & Universidade Federal do Amazonas \\
\hline Zaina Said El Hajj & Universidade Federal do Rio de Janeiro \\
\hline
\end{tabular}




\section{Origem dos Avaliadores - 2012}

\begin{tabular}{lcc}
\hline Estado & Quantidade & Percentual \\
\hline Amazonas & 4 & $3,8 \%$ \\
\hline Bahia & 3 & $2,8 \%$ \\
\hline Ceará & 7 & $6,6 \%$ \\
\hline Distrito Federal & 3 & $2,8 \%$ \\
\hline Espírito Santo & 10 & $9,4 \%$ \\
\hline Minas Gerais & 5 & $4,7 \%$ \\
\hline Paraíba & 1 & $0,9 \%$ \\
\hline Pernambuco & 4 & $3,8 \%$ \\
\hline Paraná & 6 & $5,7 \%$ \\
\hline Rio de Janeiro & 5 & $4,7 \%$ \\
\hline Rondônia & 2 & $1,9 \%$ \\
\hline Rio Grande do Sul & 4 & $3,8 \%$ \\
\hline Santa Catarina & 13 & $12,3 \%$ \\
\hline São Paulo & 38 & $35,8 \%$ \\
\hline Espanha & 1 & $0,9 \%$ \\
\hline Total & 106 & $100 \%$ \\
\hline
\end{tabular}


Instituições de Lotação dos Avaliadores - 2012

\begin{tabular}{|c|c|c|c|}
\hline Instituição & Estado & Quantidade & Percentual \\
\hline Centro Universitário Curitiba & PR & 1 & $0,9 \%$ \\
\hline Fucape Business School & ES & 8 & $7,5 \%$ \\
\hline Fundação Escola de Comércio Álvares Penteado & $\mathrm{SP}$ & 4 & $3,8 \%$ \\
\hline Fundação Getulio Vargas - SP & $\mathrm{SP}$ & 1 & $0,9 \%$ \\
\hline Fundação Instituto de Pesuisas Contábeis, Atuariais e Financeiras & $\mathrm{SP}$ & 1 & $0,9 \%$ \\
\hline Fundação Universidade Regional de Blumenau & SC & 6 & $5,7 \%$ \\
\hline Universidade Autônoma de Madrid - Espanha & Espanha & 1 & $0,9 \%$ \\
\hline Pontificia Universidade Católica de São Paulo & $\mathrm{SP}$ & 4 & $3,8 \%$ \\
\hline Receita Federal do Brasil & $\mathrm{SP}$ & 1 & $0,9 \%$ \\
\hline Universidade de Brasilia & DF & 3 & $2,8 \%$ \\
\hline Universidade de Fortaleza & CE & 1 & $0,9 \%$ \\
\hline Universidade de São Paulo & $\mathrm{SP}$ & 14 & $13,2 \%$ \\
\hline Universidade de São Paulo de Ribeirão Preto & SP & 6 & $5,7 \%$ \\
\hline Universidade do Vale do Rio dos Sinos & RS & 3 & $2,8 \%$ \\
\hline Universidade Estadual de Feira de Santana & BA & 1 & $0,9 \%$ \\
\hline Universidade Estadual de Maringá & PR & 1 & $0,9 \%$ \\
\hline Universidade Estadual de Montes Claros & MG & 1 & $0,9 \%$ \\
\hline Universidade Estadual do Oeste do Paraná & PR & 1 & $0,9 \%$ \\
\hline Universidade Estadual do Rio de Janeiro & $\mathrm{RJ}$ & 1 & $0,9 \%$ \\
\hline Universidade Estadual Paulista Julio de Mesquita Filho & SP & 1 & $0,9 \%$ \\
\hline Universidade Federal da Bahia & BA & 2 & $1,9 \%$ \\
\hline Universidade Federal da Paraíba & PB & 1 & $0,9 \%$ \\
\hline Universidade Federal de Minas Gerais & MG & 3 & $2,8 \%$ \\
\hline Universidade Federal de Pernambuco & PE & 4 & $3,8 \%$ \\
\hline Universidade Federal de Rondônia & RO & 2 & $1,9 \%$ \\
\hline Universidade Federal de Santa Catarina & SC & 7 & $6,6 \%$ \\
\hline Universidade Federal de Santa Maria & RS & 1 & $0,9 \%$ \\
\hline Universidade Federal de Uberlândia & MG & 1 & $0,9 \%$ \\
\hline Universidade Federal do Amazonas & AM & 4 & $3,8 \%$ \\
\hline Universidade Federal do Ceará & CE & 6 & $5,7 \%$ \\
\hline Universidade Federal do Espírito Santo & ES & 2 & $1,9 \%$ \\
\hline Universidade Federal do Paraná & PR & 3 & $2,8 \%$ \\
\hline Universidade Federal do Rio de Janeiro & $\mathrm{RJ}$ & 4 & $3,8 \%$ \\
\hline Universidade Municipal de São Caetano do Sul & SP & 1 & $0,9 \%$ \\
\hline Universidade Presbiteriana Mackenzie & SP & 5 & $4,7 \%$ \\
\hline Total & & 106 & $100 \%$ \\
\hline
\end{tabular}

